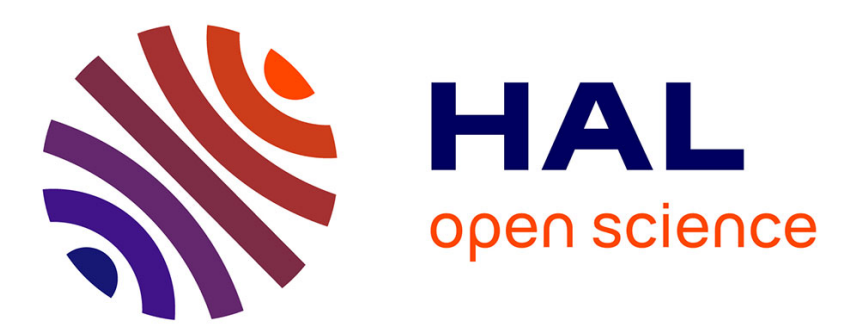

\title{
An interferometric determination of the refractive part of optical constants for carbon and silver across soft X-ray absorption edges
}

\author{
Denis Joyeux, François Polack, Daniel Phalippou
}

\section{- To cite this version:}

Denis Joyeux, François Polack, Daniel Phalippou. An interferometric determination of the refractive part of optical constants for carbon and silver across soft X-ray absorption edges. Review of Scientific Instruments, 1999, 70 (7), pp.2921-2926. hal-00867750

\section{HAL Id: hal-00867750 \\ https://hal-iogs.archives-ouvertes.fr/hal-00867750}

Submitted on 30 Sep 2013

HAL is a multi-disciplinary open access archive for the deposit and dissemination of scientific research documents, whether they are published or not. The documents may come from teaching and research institutions in France or abroad, or from public or private research centers.
L'archive ouverte pluridisciplinaire HAL, est destinée au dépôt et à la diffusion de documents scientifiques de niveau recherche, publiés ou non, émanant des établissements d'enseignement et de recherche français ou étrangers, des laboratoires publics ou privés. 


\title{
An interferometric determination of the refractive part of optical constants for carbon and silver across soft $x$-ray absorption edges
}

\author{
Denis Joyeux ${ }^{\mathrm{a})}$ \\ Laboratoire Charles Fabry-IOTA, BP147-91403 Orsay Cedex, France \\ François Polack \\ Laboratoire pour l'Utilisation du Rayonnement Electronique (LURE), Campus d'Orsay, bat 209d, \\ 91405 Orsay cedex, France \\ Daniel Phalippou \\ Laboratoire Charles Fabry-IOTA, BP147-91403 Orsay Cedex, France
}

(Received 27 January 1999; accepted for publication 15 April 1999)

\begin{abstract}
Interferometric, direct determinations of the $f_{1}$ scattering factor near absorption edges in the soft $\mathrm{x}$-ray range is demonstrated. The interferometric system, which is based on wave front division (no beam splitter) with plane mirrors only, produces a linear fringe pattern. The principle consists in direct measuring of the fringe shift occurring upon insertion of a sample into one interferometer arm, by means of a dedicated detection system. This provides the optical thickness, which in turn gives the $f_{1}$ factor, knowing either the sample mass per unit surface, or the sample thickness and density. With the sample being probed in transmission under near normal incidence, the determination of $f_{1}$ is not perturbed by the absorption part of the complex scattering factor. Therefore, $f_{1}$ data obtained here can be said new and independent with respect to those obtained previously, in the sense that they are obtained from a new, purely experimental technique, and are neither deduced from nor perturbed by absorption. The interferometer design used can be implemented in a very large spectral range. For demonstrating the ability of this interferometric system to provide such new $f_{1}$ data, a thin free-standing carbon foil near the $\mathrm{K}$ edge (from 4.1 to $4.65 \mathrm{~nm}, 302-267 \mathrm{eV}$ ), and a membrane-supported silver layer near an M edge (from 2.6 to $3.8 \mathrm{~nm}$, 477-326 eV) were used as test objects. (c) 1999 American Institute of Physics.
\end{abstract}

[S0034-6748(99)04807-8]

\section{INTRODUCTION}

In the soft x-ray to vacuum ultraviolet range as in the optical domain, the ability to determine the complex index of refraction (or its microscopic counterpart the complex scattering factor) is a major issue in a wide field of the physical sciences. These fields are related mainly to material science studies, in which the material is probed through the photonmatter interaction, but also to the emerging domain of $\mathrm{x}$-ray optical components, namely multilayers and diffractive Fresnel zone plates (FZP) lenses. Despite this obvious importance, no direct experimental method to access the real part $n=1-\delta$ of the complex index (or the $f_{1}$ scattering factor) exists up to now. More precisely, determinations are made essentially in two ways. (1) One is the inversion of the Kramers-Kronig relations from a set of absorption measurements; (2) the second is the computation of $\delta$ from angular plots of the energy reflectivity of the material, or of multilayers containing the material, through the complex Fresnel reflection formula. Both determinations suffer of the importance of the index absorption term $\beta$, relative to the refractive term $\delta$, and/or of computational problems. Although these determinations may be fair to good, far from the absorption edges, it is well known that they are questionable in

${ }^{\text {a)} E l e c t r o n i c ~ m a i l: ~ d e n i s . j o y e u x ~ @ i o t a . u-p s u d . f r ~}$ these resonance region, the most interesting ones for physical studies. ${ }^{1,2}$ Besides, these determinations always need to gather a large set of experimental data, even to obtain one single index value. This makes it difficult to study "real" materials, i.e., those which depend on the preparation conditions, which is in fact the general case and may be of importance, as for example in the case of multilayer fabrication.

Therefore, any experimental method to directly access $\delta$ without involving a significant contribution of the $\beta$ term should be welcome, especially if it also works in the resonance regions and does not involve a delicate computational process over a large data set. As indeed interferometry is the choice method to access an optical thickness, and after a pioneering, difficult experiment reported by Aoki, ${ }^{3}$ we have proposed ${ }^{4}$ to realize such a direct determination by measuring the optical phase shift that an $\mathrm{x}$-ray wave front undergoes upon transmission by a thin sample, under quasinormal incidence. ${ }^{5}$ Since then, we have reported the test of a dedicated interferometric system, using photographic recording of interferograms at $4.4 \mathrm{~nm},{ }^{6}$ and have presented qualitative ${ }^{7}$ or partial ${ }^{8}$ results for evaporated carbon at the K edge. In this article, we report and discuss the quantitative determinations that have been performed for a carbon sample across K edge and a silver sample in the region of $\mathrm{M}$ edge with our latest setup, using a direct reading on the fringe shift in the $\mathrm{x}$-ray fringe pattern. ${ }^{8}$ 


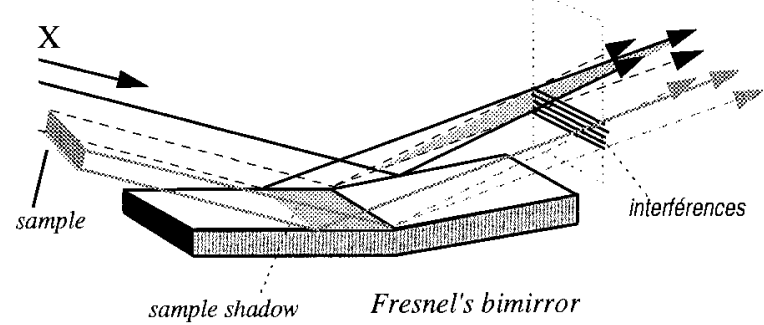

FIG. 1. Basic design of the Fresnel mirror interferometer. The sample is inserted into the incident beam in such a way it intercepts only that part of the beam which is reflected by one of the mirrors. The distance from sample to mirror ridge is $50 \mathrm{~mm}$, small enough to keep the spread out of the sample beam onto the reference mirror (due to Fresnel diffraction) negligible.

During the preparation of this article, we were informed of the article by Dambach et al., ${ }^{9}$ reporting results also obtained by two-wave interferometry from carbon at the $\mathrm{K}$ edge, with an alternative interferometric technique, namely the use of a coherent dual undulator source.

\section{THE INTERFEROMETER}

In any two beams' interferometer, introducing a transmitting sample in one of the arms shifts the fringe pattern according to $x^{(s p)}=e \delta / \lambda$, where $x^{(s p)}$ is the shift expressed in fringe spacing units, $\lambda$ the wavelength, and $e$ the sample thickness. This provides directly the optical thickness difference ( $\delta e$ ) with respect to the other arm (ideally the vacuum), which is the important quantity in some optical devices (e.g., Fresnel zone plates). Noteworthy, the knowledge of the physical thickness is required for index determinations (which are needed for multilayer design), but only the mass per unit surface (which can be measured directly and accurately during the deposition process) is needed when the $f_{1}$ scattering factor is desired.

As we use an almost identical interferometric system as described in Refs. 2 and 3, we shall just recall briefly its design, and give the geometrical parameters of interest. We use the classical ${ }^{10}$ Fresnel's bimirror configuration as depicted on Fig. 1. It is illuminated at $3^{\circ}$ grazing incidence by a synchrotron radiation (SR) beam, from the undulator SU7 of the super ACO facility (Orsay, France). Of course, any synchrotron source with an at least comparable spectral luminance is suitable. The actual source for the interferometer is the exit slit of a monochromator. It is over $10 \mathrm{~m}$ upstream the mirrors, so that the incoming wave is nearly a plane wave front. The tilt between mirrors is 1.37 arcmin, providing two beams which overlap inside a wedge shaped region with an angle $2 \alpha=2.74$ arcmin. The temporal and spatial coherence of the input beam are adjusted (both through the entrance and exit slit widths adjustments) to provide well contrasted fringes. The detector is placed $500 \mathrm{~mm}$ downstream the mirrors' common edge. With these parameters, the threedimensional (3D) fringe pattern consists in parallel planes, spaced by $5.5 \mu \mathrm{m}$ at $\lambda=4.4 \mathrm{~nm}$. At $500 \mathrm{~mm}$, the interference pattern contains about 70 linear fringes inside the geometrical shadow limits, and it is modulated by the Fresnel diffraction arising in both reflected beams from the bimirror ridge.
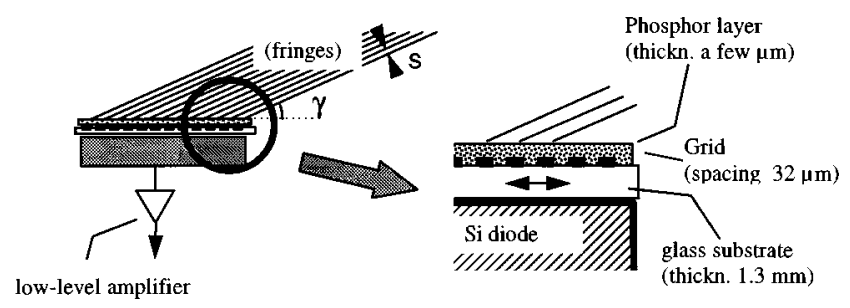

FIG. 2. Principle of the detection by moiré. The beams intersecting in the interference region are intercepted by the detector plane, under grazing incidence $\gamma$. Therefore, the phosphor layer produces a visible fringe pattern with spacing $s / \sin \gamma$. This pattern is screened by the binary grid, producing a moire pattern. The total flux in that pattern is detected by a single silicon diode. The phosphor/grid/detector assembly can be translated as indicated by arrow.

The practical realization is depicted in some detail in Ref. 8. It was made so as to fulfill two basic principles: produce a "solid" single optical part, that cannot be impaired by thermal drifts or vibrations, and work very close to the zero path difference (i.e., with the zero order fringe located inside the interference pattern), in order to minimize the necessary temporal coherence. The transmitting sample is introduced in such a way that one of the mirrors only is shadowed (sample arm) and not the other (reference arm).

\section{DETECTION PRINCIPLE AND SYSTEM ADJUSTMENT}

\section{A. Principle}

In previous experiments, we used a simple photographic detection, followed by a computer processing of the fringe patterns. ${ }^{6}$ This simple system provided the first direct experimental demonstration of the fact that carbon index crosses 1 near the $\mathrm{K}$ edge, ${ }^{8}$ but it is heavy and lengthy to operate, and it cannot be used at any wavelength. We have therefore designed and built ${ }^{8}$ a dedicated optoelectronic detection system, for a direct and quick reading of the fringe shift. This detection system is based on the production and detection of a moire pattern between the "aerial" $x$-ray fringes and a binary grid. Provided the grid is adjusted in such a way that the fringes match its spacing and orientation, the moire consists of a uniform pattern (zero spatial frequency), and the total flux transmitted by the grid is modulated periodically, with respect to the fringes-to-grid relative position. Therefore, monitoring the grid position, and tracking an extremum of the moire signal allows to measure with an excellent sensitivity any change in the pattern/grid relative position, i.e., any shift of the fringe pattern.

\section{B. Practical implementation and operating procedure}

In practice, the $x$-ray fringe pattern is first converted into a visible fringe pattern by a thin layer of very fine phosphor (Fig. 2). This layer is deposited onto a mask consisting of a binary grating etched by microlithographic techniques (for accuracy) into a standard chromium mask. The grid orientation relative to the fringe direction in the grid plane can be adjusted for parallelism. In addition, the plate is illuminated by the $\mathrm{x}$-ray beam under a grazing angle $\beta$, in such a way that the visible fringe pattern has a spacing of exactly $32 \mu \mathrm{m}$, 


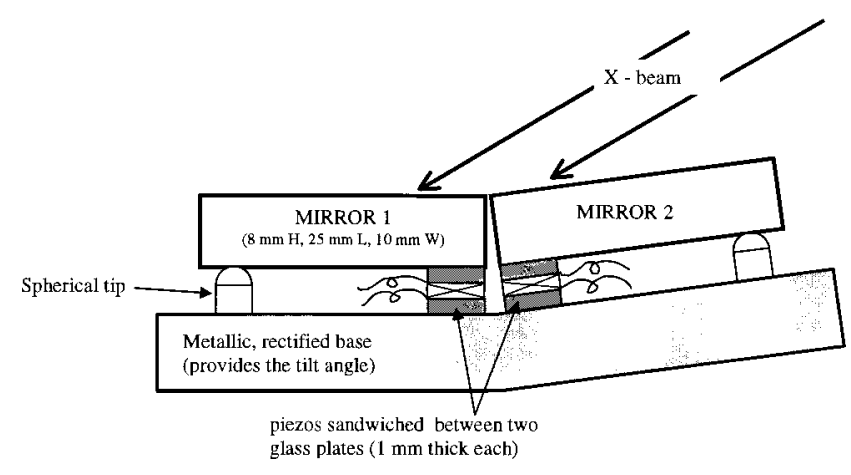

FIG. 3. The mirror system assembly showing the piezoelectric parts inserted for phase modulation. The different parts are rigidly bound by using drops of optical UV bond, at selected positions (see Ref. 8 for details on the bonding technique and the required mounting accuracy). As only one piezo is placed under each mirror, each mirror rotates around the fixed points defined by the spherical tips. However, provided that the piezo are driven with the same sinusoidal amplitude but opposite phase, the mirrors' tilt angle and therefore the fringe spacing are kept constant. In other words, the wave fronts reflected from the edge region undergo a phase shift and a small rotation, resulting in only a fringe shift, which is just what is desired. A phase modulation of $\lambda / 2$ at $\lambda=4 \mathrm{~nm}$ is obtained with peak-to-peak amplitudes of $10 \mathrm{~nm}$ (wave fronts with $3^{\circ}$ grazing incidence), producing a halffringe shift. With these conditions, and the fixed point at $20 \mathrm{~mm}$ from the piezo, the additional rotation is about $0.5 \mu \mathrm{rad}$, i.e., an additional fringe shift of $1 / 20$ of the fringe spacing, $500 \mathrm{~mm}$ downstreams.

the spacing of the grid. As the fringe spacing changes with the $\mathrm{x}$-ray wavelength, the grazing angle is adjustable, to make the system tunable in wavelength. $\beta$ is about $10^{\circ}$ at $\lambda=4.4 \mathrm{~nm}$. Finally, the phosphor-grid plate can be translated parallel to its own plane and perpendicular to the grating lines, and the grid position is monitored by a high sensitivity $(0.01 \mu \mathrm{m})$ position sensor. A single photodiode integrates the flux transmitted by the grating through the glass plate, giving the moire signal, averaged on the whole fringe pattern.

In order to get a high sensitivity in the detection of the moire maximum, it is wise to use some modulation method. We use a true phase modulation of the interferometer, by means of piezoelectric plates inserted between the mirrors and their common mount, as depicted on Fig. 3. No accuracy is required for these piezo devices, only a fair symmetry of both piezos and the stability of the average position during the time of one measurement $(\approx 1 \mathrm{mn}$, see below). The latter is kept as good as possible by working with no direct-current (dc) excitation, and by the symmetrical design of the modulation system. The phase modulation amplitude is adjusted to about $\lambda / 2$ peak-to-peak, producing a half-pitch $p$ - $p$ vibration of the fringe pattern.

With this configuration, the key signal is the time modulation of the moire signal which is synchronous with the mirror oscillation. The moiré modulation is detected by means of a lock-in amplifier with a narrow bandwidth $(1 \mathrm{~s}, 6$ $\mathrm{dB}$ roll off to $3 \mathrm{~s}, 12 \mathrm{~dB}$ output filter, depending on the signal level). The monitoring of the fringe/grid position is obtained by tracking the zeros of the synchronous signal (i.e., extrema of the dc moire signal), by means of the translation of the grid.
This system must be adjusted prior to operation, in such a way that the fringe pattern and the grid are aligned and tuned. In the present state of the system, systematic exploration of the tuning parameters is necessary, until the lock-in detects a synchronous signal. Then, this signal can easily be maximized. As a matter of fact, a good mechanical prealignment, and the use of the zero order fringe obtained with wide spectrum illumination (monochromator zero order) are enough to restrict the domain of exploration, in such a way that this procedure lasts no longer than about $15 \mathrm{~min}$. This alignment remains effective as long as the sample is not removed and the wavelength is not changed. No large drift was experienced even from day to day. When changing the wavelength, only a (predictable) change of the grazing angle on the phosphor-grid plate must be performed, as explained in the previous section.

Once the preliminary adjustments are done, the measurement procedure consists in the following: (1) without the sample inserted, adjust the grid position to zero the amplitude of the moire time modulation, and read the position $x_{1}$ of the grid; (2) after inserting the sample, retrieve a zero moire signal by translating the grid, and read again the new position $x_{2}$. Then, the optical thickness increment $\Delta t$ is such that $\left(x_{2}-x_{1}\right) /$ pitch is the fractional part of $\Delta t / \lambda$. The integer part (which should usually be 0 or 1 ) can be determined by continuity and/or from known data far from edges. The sign of the increment can be derived either from a priori data, or from the sign of the shift and the analysis of the interferometer geometry.

\section{Discussion}

The main properties of this detection system are its very large sensitivity to shifts, which is scaled by the spatial period, and its spatial frequency filtering capability. As a matter of fact, it is equivalent to a lock-in detector in the spatial domain, being used as a phase detector yielding the relative phase of the grid (the reference spatial frequency) to the $\mathrm{x}$-ray fringes (the signal). In particular, if the grid lines width are equal to half its period, it provides a good rejection of the second harmonic pattern, which occurs if spectral pollution by the grating 2 nd harmonic exist in the beam line monochromator (a usual problem of monochromators in this spectral range).

This principle was first tested in visible light on an optical bench, ${ }^{11}$ with similar geometrical parameters (i.e., spacing and fringe number, but without the phosphor layer). We found that a sensitivity of about $\lambda / 800$ was achievable in these conditions. It is worth mentioning that the $X$-to-visible conversion is not mandatory. On the contrary, a direct detection by a silicon diode having its surface masked by a grid (using microlithographic masking and etching technique) would be much more efficient in terms of the dc and modulation levels, and should allow a gain in sensitivity that we estimate to be at least one order of magnitude. At this point of the study, however, the conversion was preferred because it allows much more flexibility in the setup realization, and a much lower price. 


\section{EXPERIMENTS AND RESULTS}

\section{A. Consistency of wavelength scales and spectral width}

As data were obtained during different runs of the synchroton source, it was necessary to determine the mutual shift of the wavelength scales of the different series. This was made by watching during every run the position of the spectral dip arising from (small) absorption by the carbon pollution on optical surfaces in the beam line. Using one particular spectrum as a common reference, and applying a simple correlation algorithm, we have obtained an accuracy better than $0.002 \mathrm{~nm}$ on shifts (regardless of the spectral resolution). As for the absolute recalibration, we have assumed that the main absorption edge position of our carbon samples is identical to the tables value, i.e., $284.2 \mathrm{eV}$ or 4.36 $\mathrm{nm}$, and we have shifted all experimental wavelength scales accordingly. As a matter of fact, this correction shifts the minimum of the beamline absorption dip to $285.1 \mathrm{eV}$. Considering the low spectral resolution (see below), this is close enough to the position generally admitted $\left(285.5 \mathrm{eV}, \pi^{*}\right.$ resonance). We have assumed that this recalibration procedure is still good enough at $\lambda=3 \mathrm{~nm}$.

As a matter of facts, experiments were done with a quite limited spectral resolution, namely $\lambda / \Delta \lambda \approx 125$ and 250 for carbon and $\lambda / \Delta \lambda \approx 120$ for silver, because it was necessary to open the input slit of the monochromator to keep the detected flux level large enough. (This is related to the available "coherent flux" which is determined by the source spectral luminance, the wavelength, and the spectral width $^{12}$ ). The 3 rd generation synchrotron sources offer a larger spectral luminance, and would allow working with much smaller spectral width, or, alternatively, a much smaller detection noise and the same width.

\section{B. The samples}

The experiments reported in this article concerned carbon in the range $\lambda=4.1$ to $4.65 \mathrm{~nm}(302-267 \mathrm{eV})$ and silver in the range $\lambda=2.6-3.8 \mathrm{~nm}(477-326 \mathrm{eV})$.

The carbon sample was a free standing foil of evaporated carbon, supplied by Goodfellow (its index was therefore measured with respect to vacuum). The surface mass is $52.0 \mu \mathrm{g} / \mathrm{cm}^{2}$ (i.e., a thickness of $0.26 \mu \mathrm{m}$ for a nominal density of 2). The size parallel to fringes is $4 \mathrm{~mm}$. The sample was not characterized with respect to its atomic/ molecular structure (see discussion below).

The silver sample consists of a layer deposited on half the window of a silicon nitride membrane $50 \mathrm{~nm}$ thick. It is therefore assumed that the region of the membrane which is involved in the interferogram building (i.e., $380 \mu \mathrm{m}$ on both sides of the layer edge) is uniform enough not to disturb the interferogram of the silver layer. (As a matter of fact, it was checked that shifting the membrane by $380 \mu \mathrm{m}$ from the no-sample condition toward the no-sample side produces no detectable phase shift). The layer thickness is $150 \mathrm{~nm} \pm 1 \%$, and the height parallel to fringes is about $5 \mathrm{~mm}$. The layer density is estimated to be $10.2 \mathrm{~g} / \mathrm{cm}^{3}$ (bulk density is 10.5) by fitting data from grazing incidence hard $x$-ray $(\lambda$ $=0.1 \mathrm{~nm}$ ) reflectivity measurements.

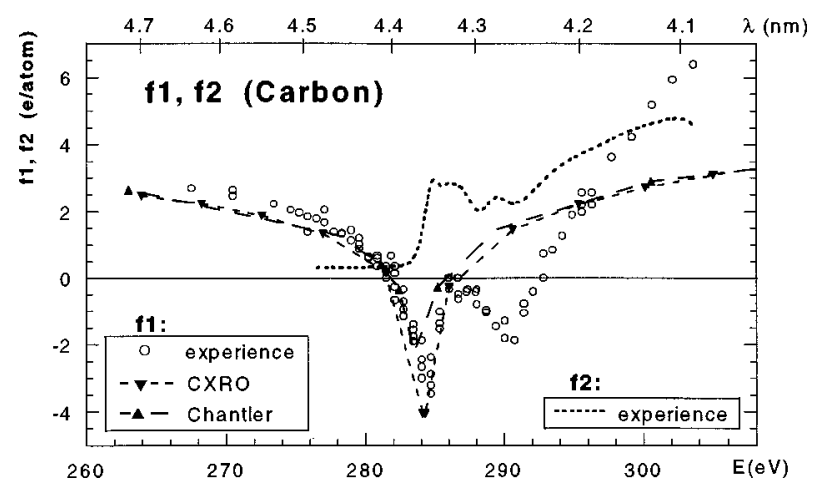

FIG. 4. Measured and tabulated scattering factors for carbon. See the text.

\section{Results}

Experimental results are displayed in terms of both scattering factors $f_{1}$ and $f_{2}$ on Figs. 4 and 5. Also shown are the corresponding data extracted from two particular tables: the CXRO table ${ }^{13}$ and the table published by Chantler in $1995 .{ }^{14}$ It may be useful to mention the order of magnitude of the corresponding fringe shifts: a shift of 1 fringe would be produced by $f_{1} \approx 20.5 \mathrm{e} / \mathrm{atom}$ at $\lambda=4.2 \mathrm{~nm}$ for the carbon sample, and $f_{1} \approx 79 \mathrm{e} /$ atom at $\lambda=3.2 \mathrm{~nm}$ for the silver sample. It is therefore seen that the shifts were most generally smaller than $1 / 4$ fringe in these experiments, and the problem of the integer part of the shift (in fringe spacing units) never occurred.

The absorption of samples was measured with the same setup by recording the total transmitted signal on the interferometric detector (grid and fringes being unmatched) once
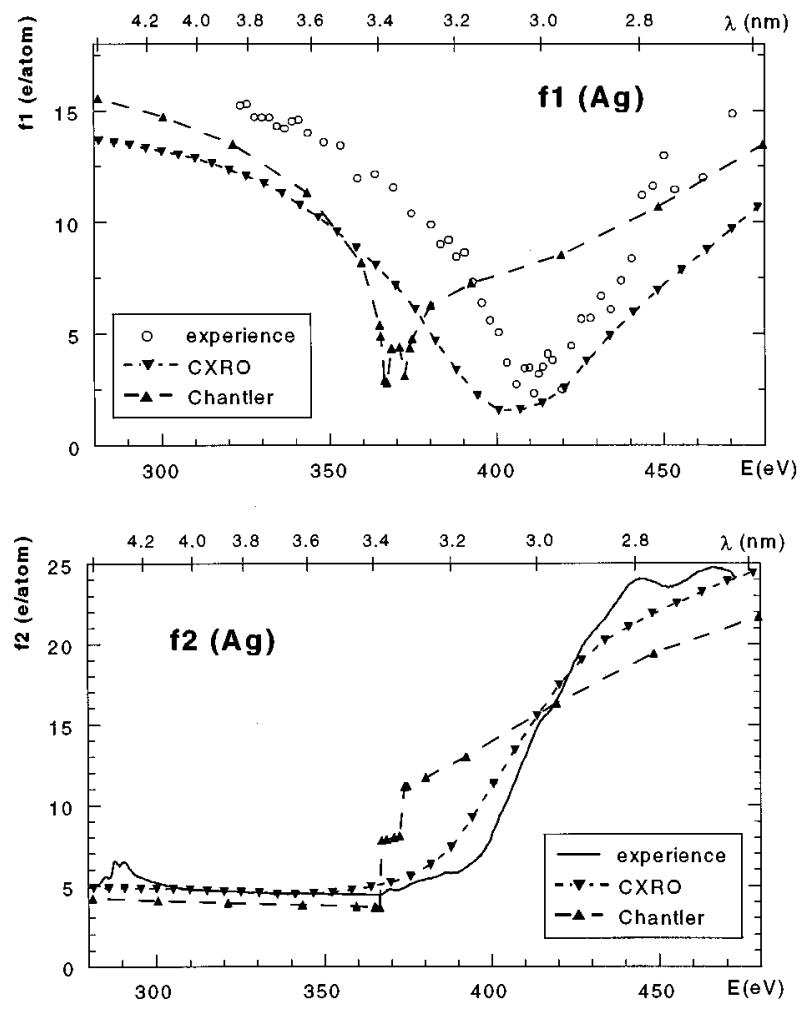

FIG. 5. Measured and tabulated scattering factors for silver. See the text. 
with no sample in the field, once with the sample covering both mirrors. This allows elimination of all spectral variations of the transmitted signal that arise from anything but the sample. In the same time, an auxiliary detector (an x-ray UDT silicon diode) provided a reference signal, to allow for the compensation of the synchrotron beam time-decrease.

\section{DISCUSSION}

\section{A. Experimental accuracy}

Experimental data are submitted to several error causes, which have their origins in the experience realization, and, for one term, in the very principle of the method. This latter term is related to the wave front division principle, which makes the fringe pattern a limited and diffraction modulated set of linear fringes. It is therefore not a pure sinusoidal pattern, in the sense of the spatial frequency spectrum. Taking realistic parameter values, computer simulations of the moire detection process show that the error introduced by the moire detection system in the shift measurement is never greater than $1 / 250$ of the spacing in the worst case, and generally much better.

Experimental error terms have their main origin in the detection noise. For each reading of the grid position, the detection noise sets a precision limit that we estimate to be about $0.05-0.1 \mu \mathrm{m}$, depending on the signal level. Therefore, the shift reading noise error is $0.07-0.15 \mu \mathrm{m}$, i.e., $\lambda / 400-\lambda / 200$ for the optical thickness. The same absolute reading error applies to the grid spacing value, because the position sensor was calibrated by measuring the distance between two successive moire extremums (which should be the grid spacing, by principle). As this value is a multiplicative factor in the fringe shift data processing, it introduces in the final result a relative error which should be better than 1/200. It is therefore not the main error term when the shift is small compared to the spacing. Finally, an additional random error in the shift reading was found, because of a lack of rigidity of the setup. Although this error was partly removed by repeated position readings, it is responsible for the fluctuating appearance of the $f_{1}$ data sets, especially for silver. This term should be eliminated before realization of further measurements.

In summary, the accuracy of the experimental data presented is limited by an experimental random error term, which should be removed for further operations. Apart from this term, the absolute accuracy on $\delta$ or $f_{1}$, is essentially given by the absolute error on the shift determination, which should be not greater than $\approx($ spacing/200). According to Sec. IVC this represents an absolute accuracy of $\approx 0.1$ e/atom on $f_{1}$ or $8 \times 10^{-5}$ on $\delta$ for the carbon sample, and 0.4 e/atom or $1 \times 10^{-4}$ for the silver sample. This should not suggest to use very thick samples, because the moiré signal, and therefore the fringe contrast, must be kept to a reasonable level, and a tradeoff must be accepted. The thicknesses we have used for $\mathrm{C}$ and $\mathrm{Ag}$ samples can be considered reasonable, although the fringe shift was almost always smaller than a half fringe.

\section{B. Physical significance of $f_{1}$ data}

As for the physical significance of the data presented, it must first be pointed out that the three sets of data are not exactly comparable (and comparison was not the main goal of this series of experiments). Whereas our data are obtained from a particular "real" sample, those of CXRO are deduced by averaging various experimental data (for $f_{2}$ ) and applying Kramers-Kronig based computations (for $f_{1}$ ), and those of Chantler are ab initio calculations based on a modelization of the (ideal) material behavior. Therefore, it is most likely that the three data sets cannot be identical, especially in resonance regions. This is true for silver, the nonstandard behavior of which is well known even in the visible range, and for carbon, in which the atomic structure may vary strongly due to the fabrication technique and the history of the sample. In any case, although we claim that this kind of measurements might change or improve the tabulated data presently in use, this is not the case for the particular data presented, which are still too sparse and concern particular samples.

However, it is remarkable that the data presented for silver are quite close (at least in shape) to the CXRO tabulated data. Significant differences might be the sharpest dip of $f_{1}$, which corresponds to the steepest edge for $f_{2}$. As for the carbon, it is found that, on the high energy side of the edge, our data are quite different from tables and those presented in Ref. 9 (for $f_{1}$ as well as for $f_{2}$ ). In particular, Ref. 9 results show clearly the presence of a $\pi^{*} \mathrm{C}-\mathrm{C}$ resonance at $284.5 \mathrm{eV}$, while it seems that there is no particular structure in front of the edge in our data. This would mean an amorphous structure, but our sample shows also a strong after-edge feature $\left(f_{1}<0\right.$, corresponding to a value of $f_{2}$ well below the value given by all tables), while a similar structure seems to be much weaker on Ref. 9 sample. All this emphasizes strongly the need of being able to measure independently $f_{1}$ for real materials, as well for x-ray optics applications for material sciences studies.

It is obvious that independent determinations of $f_{1}$ and $f_{2}$ should be cross checked with respect to Kramers-Kronig relations in the end. This was not done here essentially because the spectral range of the new data was not wide enough (an advantage of interferometric method under other respects...). Moreover, it is not obvious (for the moment) whether $f_{1}$ or $f_{2}$ or both can be considered as accurate enough, so that cross-checking is reliable. After all, $f_{1}$ determinations from $f_{2}$ are generally considered as questionable in the resonance regions.

\section{Further improvements and application}

As mentionned, the experimental system can be improved with respect to its reliability and ease of use. Also, it is desirable to improve the spectral resolution. As it is presently limited by the photon budget, this can be obtained both by using a higher luminance source (last generation SR source) and by using a direct $\mathrm{x}$-ray detector as explained.

More generally, these experimental principles can be applied in a wide spectral range from EUV to soft $x$ rays, with a suitable adjustment of its parameters. Therefore, such scat- 
tering factor measurements should provide a new independent source of data, allowing to cross-check other data sources, and to improve the modeling of atomic interaction in absorption edge regions. The introduction of interferometric techniques for phase measurement might also be beneficial to surface science based on reflectivity measurements. It is likely that the introduction of phase data, i.e., the measurement of the complex reflectivity, should improve significantly the determination of the permittivity matrix from the resolution of the Fresnel equations.

\section{ACKNOWLEDGMENTS}

A preliminary but important part of this work was made by Jan Svatos during his thesis, with the help of Ben Bradshaw, an Erasmus student from Southampton University. We are also indebted to our colleagues Jean-Pierre Chauvineau and Françoise Bridou (Institut d'Optique) who have realized and calibrated the silver sample. Finally, the polishing and assembly of the soft x-ray interferometer is a masterwork, that only the skill and experience of the Institut d'Optique's optical workshop have made possible.
${ }^{1}$ C. T. Chantler, Radiat. Phys. Chem. 41, 759 (1993).

${ }^{2}$ E. Spiller, Appl. Opt. 29, 19 (1990).

${ }^{3}$ S. Aoki and S. Kikuta, Inst. Phys. Conf. Ser. 147, 49 (1986).

${ }^{4}$ F. Polack and D. Joyeux, in X-Ray Microscopy III, edited by A. G. Michette, G. R. Morrison, and C. J. Buckley (Springer, Berlin, 1992), p. 301.

${ }^{5}$ U. Bonse and M. Hart, Appl. Phys. Lett. 7, 238 (1965).

${ }^{6}$ J. Svatos, D. Joyeux, F. Polack, and D. Phalippou, Opt. Lett. 18, 1367 (1993).

${ }^{7}$ Results were first presented in: J. Svatos, D. Joyeux, F. Polack, and D. Phalippou, XRM '93 conference on X ray microcopy, Chernogolovka (Russie), 3-7 September 1993 (unpublished). This communication was accidentaly omitted from the conference proceedings, and the main result was reproduced in Ref. 8.

${ }^{8}$ D. Joyeux and F. Polack, in X-Ray Microscopy and Spectromicroscopy, edited by J. Thieme, G. Schmahl, D. Rudolph, and E. Umbach (Springer, Berlin, 1998), p. II-103.

${ }^{9}$ S. Dambach, H. Backe, Th. Doerk, N. Eftekhari, H. Euteneuer, F. Görgen, F. Hagenbuck, K. H. Kaiser, O. Kettig, G. Kube, W. Lauth, H. Schöpe, A. Steinhof, Th. Tonn, and Th. Walcher, Phys. Rev. Lett. 80, 5473 (1998).

${ }^{10}$ See, for example, M. Born and E. Wolf, Principle of Optics (Pergamon, New York, 1964), p. 262.

${ }^{11}$ J. Svatos, Ph.D. thesis, Université paris XI, Orsay, France, 1994.

${ }^{12}$ D. Joyeux, P. Jaeglé, and A. L'Huillier, in Trends in Optics, Vol 3, edited by A. Consortini (Academic, London, 1996), p. 371.

${ }^{13}$ B. L. Henke, E. M. Gullikson, and J. C. Davis, At. Data Nucl. Data Tables 54, 181 (1993).

${ }^{14}$ C. T. Chantler, J. Phys. Chem. Ref. Data 24, 1 (1995). 